\title{
EINSTEIN MANIFOLDS OF NON-NEGATIVE SECTIONAL CURVATURE AND ENTROPY
}

\author{
Gabriel P. Paternain and Jimmy Petean
}

ABSTRACT. We show that if $\left(M^{n}, g\right)$ is a closed Einstein manifold of non-negative curvature then $-\log R \leq \frac{\pi \sqrt{n-1}(n-2)}{2}$, where $R$ is the radius of convergence of the series $\sum_{i \geq 2} \operatorname{dim}\left(\pi_{i}(M) \otimes \mathbb{Q}\right) t^{i}$. If we suppose in addition that $M$ is formal then we show that:

$$
\operatorname{dim} H_{*}(M, \mathbb{Q}) \leq\left[1+\exp \left(\frac{\pi \sqrt{n-1}(n-2)}{2}\right)\right]^{n}
$$

These results are achieved by combining the classical Morse theory of the loop space with a new upper bound for the topological entropy of the geodesic flow of $g$ in terms of the curvature tensor.

\section{Introduction}

Let $\left(M^{n}, g\right)$ be a complete smooth connected Riemannian manifold. One of our main concerns here will be the study of Einstein metrics $g$ of non-negative sectional curvature. Unless $(M, g)$ is flat, the Ricci curvature $r$ must be positive and we might as well rescale the metric so that $r=g$. Therefore all the sectional curvatures satisfy $0 \leq K(P) \leq 1$. Myers' theorem asserts that $M$ is compact, has finite fundamental group and its diameter is $\leq \sqrt{n-1} \pi$. By passing to the universal covering we will usually assume that $M$ is in fact closed and simply connected.

When $M$ is four-dimensional, strong topological restrictions to the existence of such metrics were first given by N. Hitchin [18]. He proved that the Euler characteristic $\chi$ and the signature $\tau$ of $M$ must satisfy

$$
\chi \geq\left(\frac{3}{2}\right)^{3 / 2}|\tau| .
$$

This inequality was recently improved by M.J. Gursky and C. LeBrun [15] who showed that in fact one has $9 \geq \chi>15 / 4|\tau|$. This result together with Freedman's classification theorem [10] tells us that there are at most 12 homeomorphism types (which can be explicitly listed) of simply connected closed Einstein manifolds of non-negative sectional curvature. If one assumes further that $M$

Received May 24, 2000.

1991 Mathematics Subject Classification. 53C25, 53C21, 58F17, 35J15.

G.P. Paternain is on leave from Centro de Matemática, Facultad de Ciencias, Iguá 4225, 11400 Montevideo, Uruguay.

J. Petean is supported by grant 28491-E of CONACYT. 
has positive definite intersection form, then Gursky and LeBrun proved that $(M, g)$ must, up to isometry, be $\mathbb{C} P^{2}$ equipped with a constant multiple of the Fubini-Study metric. All these results rely heavily on the four-dimensional Gauss-Bonnet theorem.

Even though it is believed that simply connected closed Einstein manifolds with non-negative sectional curvature are very rare, there are basically no known topological obstructions to the existence of such metrics in dimensions $\geq 5$, except, of course, Gromov's celebrated result in [11] which gives an obstruction to the existence of metrics just with non-negative sectional curvature. Gromov's result asserts that if $M$ admits such a metric, then the sum of the Betti numbers $b_{i}$ must verify:

$$
\operatorname{dim} H_{*}(M, \mathbb{Q})=\sum_{i=0}^{n} b_{i} \leq C(n),
$$

where

$$
C(n):=((n+1) J(n))^{100^{n}}, \quad J(n):=2^{M(n)}, \quad M(n):=8^{n} 10^{n^{2}+4 n} .
$$

Clearly, the bound $C(n)$ is quite unrealistic, and Gromov himself conjectured that $C(n)$ should be just $2^{n}$.

We now state our first main result. Let $R$ be the radius of convergence of the series

$$
\sum_{i \geq 2} \operatorname{dim}\left(\pi_{i}(M) \otimes \mathbb{Q}\right) t^{i}
$$

Theorem A. Let $\left(M^{n}, g\right)$ be a smooth closed manifold of positive Ricci curvature. Suppose that we normalize $g$ so that $r \geq g$ and let $k:=\max _{P} K(P)$. Then

$$
-\log R \leq \frac{\pi \sqrt{n-1}}{2}\left((n-1) \sqrt{k}-\frac{1}{\sqrt{k}}\right) .
$$

In particular, if $\left(M^{n}, g\right)$ is a closed Einstein manifold of non-negative sectional curvature, then

$$
-\log R \leq \frac{\pi \sqrt{n-1}(n-2)}{2}
$$

We remark that to prove Theorem A it is enough to consider the case when $M$ is simply connected. Also note that the second inequality in the theorem (as well as all the corollaries below) can also be obtained just assuming the existence of a metric $g$ with $r \geq g$ and $K(P) \leq 1$ for all $P$.

A simply connected closed manifold $M$ is said to be rationally elliptic if the rational homotopy $\pi_{*}(M) \otimes \mathbb{Q}$ is finite dimensional, i.e. there exists a positive integer $i_{0}$ such that for all $i \geq i_{0}, \pi_{i}(M) \otimes \mathbb{Q}=0$. The manifold $M$ is said to be rationally hyperbolic if it is not rationally elliptic (cf. [7, 8, 13] and references therein). 
It was proved by Y. Félix and S. Halperin [6] that if $M$ is rationally hyperbolic, then the integers $\rho_{i}=\sum_{j \leq i} \operatorname{dim} \pi_{j}(M) \otimes \mathbb{Q}$ grow exponentially in $i$ (i.e. there exist $C>1$ and a positive integer $k$ such that if $i>k$ then $\rho_{i} \geq C^{i}$ ). Hence if $M$ is rationally elliptic we have $R=+\infty$ and if $M$ is rationally hyperbolic $R<1$. It follows that Theorem $\mathrm{A}$ is meaningful only if $M$ is rationally hyperbolic.

The "generic" manifold is rationally hyperbolic; rational ellipticity is a severely restrictive condition. Examples of rationally elliptic manifolds are simply connected homogeneous spaces [29], manifolds that admit a codimension one compact action [16], Dupin hypersurfaces [16] and any known manifold that admits a Riemannian metric of non-negative sectional curvature. A conjecture attributed to R. Bott states that any compact simply connected manifold that admits a metric of non-negative sectional curvature must be rationally elliptic (cf. [13]). It is known that if $M$ is rationally elliptic then $\operatorname{dim} H_{*}(M, \mathbb{Q}) \leq 2^{n}$ $[13,17]$ and hence Bott's conjecture implies Gromov's conjecture on the optimal bound for the sum of the Betti numbers of $M$.

A manifold $M$ is said to be formal if there exists a morphism of differential graded algebras from the minimal model of $M$ to $\left(H^{*}(M, \mathbb{Q}), 0\right)$ that induces an isomorphism in cohomology. The interest for this class of spaces lies in the fact that for them all the rational homotopy invariants of $M$ can be obtained from $H^{*}(M, \mathbb{Q})$. In [4], it is shown that compact simply connected Kähler manifolds are formal. Also, any manifold with dimension $\leq 6$ is formal and, more generally, any $p-1$-connected manifold of dimension $\leq 4 p-2$ is formal [25].

In Section 2 we shall explain how the results of Y. Félix and J.C. Thomas in [9] combined with Theorem A yield the following two corollaries:

Corollary 1. Let $\left(M^{n}, g\right)$ be a smooth closed Einstein manifold of non-negative sectional curvature. If $M$ is formal we have:

$$
\operatorname{dim} H_{*}(M, \mathbb{Q}) \leq\left[1+\exp \left(\frac{\pi \sqrt{n-1}(n-2)}{2}\right)\right]^{n} .
$$

Corollary 2. Let $\left(M^{n}, g\right)$ be a smooth closed Einstein manifold of non-negative sectional curvature. Suppose that $M$ is formal and $(p-1)$-connected. Then

$$
b_{p} \leq \frac{n}{p} \exp \left(\frac{p \pi \sqrt{n-1}(n-2)}{2}\right) \text {. }
$$

From Corollary 2, we obtain right away:

Corollary 3. Let $\left(M^{5}, g\right)$ be a smooth closed Einstein 5-dimensional manifold of non-negative sectional curvature. Then

$$
b_{2} \leq \frac{5}{2} \exp (6 \pi)
$$


Corollary 3 implies, for instance, that the connected sum of $k$ copies of $S^{3} \times S^{2}$ cannot admit an Einstein metric of non-negative sectional curvature for $k>$ $\frac{5}{2} \exp (6 \pi)$.

Certainly the bounds in Corollaries 1 and 3 are far better than Gromov's bound. On the other hand our bounds are not as good as those obtained by Gursky and LeBrun in [15] for four manifolds. The best lower bound for $-\log R$ in dimension four has been obtained by I. Babenko in [1]. He proved that

$$
1 / R \geq \frac{b_{2}+\sqrt{b_{2}^{2}-4}}{2} .
$$

Combining this bound with Theorem A we obtain:

$$
\frac{b_{2}+\sqrt{b_{2}^{2}-4}}{2} \leq \exp (\pi \sqrt{3})
$$

This implies that $b_{2} \leq 230$ while Gursky and LeBrun's result implies that $b_{2} \leq 7$.

The proof of Theorem A is based on ideas first introduced by M. Berger and R. Bott in [3] and further developed by N. Grossman in [12]. Given $x$ and $y$ in $M$ and $T>0$, define $n_{T}(x, y)$ as the number of geodesic arcs joining $x$ and $y$ with length $\leq T$. For each $T>0$, the counting function $n_{T}(x, y)$ is finite and locally constant on an open full measure subset of $M \times M$, and integrable on $M \times M$. If $g$ has positive Ricci curvature, i.e., $r \geq \delta g$ with $\delta>0$, then we shall see in Section 2 that the Morse theory of the loop space yields

$$
\frac{-\sqrt{\delta} \log R}{\pi \sqrt{n-1}} \leq \limsup _{T \rightarrow+\infty} \frac{1}{T} \log \int_{M} n_{T}(x, y) d y,
$$

for any point $x \in M$. The first author explained in [27] how Yomdin's theorem [30] can be used to prove that

$$
\limsup _{T \rightarrow+\infty} \frac{1}{T} \log \int_{M} n_{T}(x, y) d y \leq h_{t o p}(g)
$$

where $h_{t o p}(g)$ denotes the topological entropy of the geodesic flow of $g$. Combining (1) and (2) yields:

$$
\frac{-\sqrt{\delta} \log R}{\pi \sqrt{n-1}} \leq h_{t o p}(g)
$$

Inequality (3) is also pointed out by I. Babenko in [1, Theorem 4]. While Berger, Bott and Grossman estimated the average counting function by using Rauch's comparision theorem we shall use dynamical ideas to estimate $h_{t o p}(g)$ from above. As we shall see below our bound is better than those of Berger, Bott and Grossman and does not involve any lower bound on the sectional curvature. In Section 3 we shall prove the following result which will imply Theorem A and has independent interest. 
Theorem B. Let $\left(M^{n}, g\right)$ be a closed Riemannian manifold and let $K_{\max }$ be a positive upper bound for the sectional curvature. Then

$$
h_{\text {top }}(g) \leq \frac{n-1}{2} \sqrt{K_{\max }}-\frac{\min _{v \in S M} r(v)}{2 \sqrt{K_{\max }}},
$$

where $S M$ is the unit sphere bundle of $M$ and $r(v)$ is the Ricci curvature in the direction of $v \in S M$.

Let $k$ be a positive number such that $|K(P)| \leq k$ for all 2-planes $P$. Then, clearly $r \geq-(n-1) k g$ and hence Theorem B gives

$$
h_{\text {top }}(g) \leq \frac{n-1}{2} \sqrt{k}+\frac{n-1}{2} \sqrt{k}=(n-1) \sqrt{k} .
$$

The latter inequality, which is certainly weaker than that of Theorem B, was first proved by A. Manning in [21]. The upper bound for $h_{t o p}(g)$ in Theorem B is probably the best that one can obtain in terms of the $C^{0}$-norm of the curvature tensor. Note that the bound becomes sharp for all the space forms (Manning's bound is not sharp for positively curved space forms). Also observe that there is no hope to obtain an upper bound for $h_{t o p}(g)$ purely in terms of bounds of the Ricci curvature. Indeed, the $K 3$ surface admits Ricci flat metrics, but $h_{\text {top }}(g)>0$ for any $C^{\infty}$ metric $g$ since the $K 3$ surface is rationally hyperbolic $[26,27]$ (any simply connected four-manifold with second Betti number strictly bigger than two is rationally hyperbolic).

We conclude this introduction by comparing the upper bound in Theorem B with the bounds obtained by Berger and Bott in [3] and Grossman in [12]. Grossman's bound gives Berger and Bott's bound if one assumes that the manifold has positive sectional curvature. The first important difference between their bounds and ours is that ours does not involve any lower bound on the sectional curvatures; this is certainly an advantage if one is interested in upper bounds for $-\log R$ as in Theorem A. But even in the case of manifolds of nonnegative sectional curvature our bound is sharper as we now explain. To make the comparison easier suppose that our Riemannian metric $g$ has $0 \leq K(P) \leq 1$ for all 2-planes $P$. In this case our Theorem B gives

$$
h_{\text {top }}(g) \leq \frac{1}{2}\left(n-1-\min _{v \in S M} r(v)\right) .
$$

Under the same hypotheses on $g$ the inequality in Proposition 5.1 in [12] yields

$$
\limsup _{T \rightarrow+\infty} \frac{1}{T} \log \int_{M} n_{T}(x, y) d y \leq \frac{2(n-1)}{\pi} \log \left(2+\frac{\pi}{2}\right) .
$$

which is certainly weaker than our bound even ignoring the term involving the Ricci curvature since $\frac{2}{\pi} \log \left(2+\frac{\pi}{2}\right)$ is aproximately 0.8103 . 


\section{Proof of Theorem A and Corollaries 1 and 2}

2.1. Proof of Theorem A. As in the introduction, given $x$ and $y$ in $M$ and $T>0$, let $n_{T}(x, y)$ be the number of geodesic arcs joining $x$ and $y$ with length $\leq T$. For each $T>0$, the counting function $n_{T}(x, y)$ is finite and locally constant on an open full measure subset of $M \times M$, and integrable on $M \times M[3,26]$.

Suppose now that the points $x$ and $y$ are not conjugate along any geodesic connecting them. Let $\Omega(x, y)$ be the space of piecewise smooth paths $\alpha:[0,1] \rightarrow$ $M$ with $\alpha(0)=x$ and $\alpha(1)=y$. Given a non-negative integer $q$, let $i_{q}(x, y)$ be the number of geodesic arcs from $x$ to $y$ with index $\leq q$. The Morse inequalities applied to the energy functional on $\Omega(x, y)$ give right away [24]:

$$
\sum_{i=0}^{q} b_{i}(\Omega(x, y)) \leq i_{q}(x, y) .
$$

It follows from the proof of Myers' theorem that if the Ricci curvarture $r$ satisfies $r \geq \delta g$ for $\delta>0$, then any geodesic arc between $x$ and $y$ with length at least $\pi \sqrt{\frac{n-1}{\delta}} q$ has index at least $q$. Hence for any non-negative integer $q$ we have:

$$
i_{q}(x, y) \leq n_{\pi \sqrt{\frac{n-1}{\delta}} q}(x, y) .
$$

Combining (4) and (5) we obtain

$$
\sum_{i=0}^{q} b_{i}(\Omega(x, y)) \leq n_{\pi \sqrt{\frac{n-1}{\delta}} q}(x, y)
$$

and integrating this inequality with respect to $y$ gives:

$$
\sum_{i=0}^{q} b_{i}(\Omega(x, y)) \leq \frac{1}{\operatorname{Vol}(M)} \int_{M} n_{\pi \sqrt{\frac{n-1}{\delta}} q}(x, y) d y,
$$

and hence

$$
\limsup _{q \rightarrow+\infty} \frac{1}{q} \log \sum_{i=0}^{q} b_{i}(\Omega(x, y)) \leq \pi \sqrt{\frac{n-1}{\delta}} \limsup _{T \rightarrow+\infty} \frac{1}{T} \log \int_{M} n_{T}(x, y) d y .
$$

This argument and the last inequality are taken from $[3,12]$.

Now let $R_{\Omega}$ be the radius of convergence of the Poincaré series:

$$
\sum_{i \geq 0} b_{i}(\Omega(x, y), \mathbb{Q}) t^{i} .
$$

Since this series always has infinitely many non-zero coefficients we clearly have:

$$
-\log R_{\Omega}=\limsup _{q \rightarrow+\infty} \frac{1}{q} \log \sum_{i=0}^{q} b_{i}(\Omega(x, y), \mathbb{Q}) .
$$

On the other hand Babenko showed in [2] that if $M$ is rationally hyperbolic, then $R=R_{\Omega}$ where $R$ is the radius of convergence of:

$$
\sum_{i \geq 2} \operatorname{dim}\left(\pi_{i}(M) \otimes \mathbb{Q}\right) t^{i} .
$$


Using (6) we obtain inequality (1) in the introduction, namely:

$$
\frac{-\sqrt{\delta} \log R}{\pi \sqrt{n-1}} \leq \limsup _{T \rightarrow+\infty} \frac{1}{T} \log \int_{M} n_{T}(x, y) d y .
$$

In [27], the first author explained how Yomdin's theorem [30] can be used to prove that (see also [26]):

$$
\limsup _{T \rightarrow+\infty} \frac{1}{T} \log \int_{M} n_{T}(x, y) d y \leq h_{t o p}(g)
$$

where $h_{t o p}(g)$ denotes the topological entropy of the geodesic flow of $g$. Combining (7) and (8) yields:

$$
\frac{-\sqrt{\delta} \log R}{\pi \sqrt{n-1}} \leq h_{t o p}(g)
$$

Theorem A is an immediate consequence of inequality (9), Theorem B and the following observation: if $M$ admits an Einstein metric $g$ of non-negative sectional curvature, then we can rescale $g$ so that $r=g$ and $0 \leq K(P) \leq 1$.

2.2. Proof of Corollary 1. We will use the following result of Y. Félix and J.C. Thomas [9]:

Theorem 2.1. Suppose that $M$ is formal and rationally hyperbolic and let $P_{M}$ be the Poincaré polynomial of $H_{*}(M, \mathbb{Z})$. Write $P_{M}(t)=\prod_{i=1}^{n}\left(t-z_{i}\right)$. Then

$$
R \leq \min _{1 \leq i \leq n}\left|z_{i}\right|
$$

Corollary 2.2. Let $M^{n}$ be a closed formal rationally hyperbolic manifold. Then

$$
\operatorname{dim} H_{*}(M, \mathbb{Q}) \leq\left(1+\frac{1}{R}\right)^{n}
$$

Proof. By Poincaré duality if $z$ is a root of $P_{M}(t)$ then $1 / z$ is also a root of $P_{M}(t)$. Hence if we write

$$
P_{M}(t)=\prod_{i=1}^{n}\left(t-z_{i}\right)
$$

it follows that

$$
\min _{1 \leq i \leq n}\left|z_{i}\right|=\frac{1}{\max _{1 \leq i \leq n}\left|z_{i}\right|}
$$

Let us set for brevity $A:=\max _{1 \leq i \leq n}\left|z_{i}\right|$. From (10) we get:

$$
b_{i} \leq\left(\begin{array}{c}
n \\
i
\end{array}\right) A^{i}
$$

hence

$$
\operatorname{dim} H_{*}(M, \mathbb{Q}) \leq \sum_{i=0}^{n}\left(\begin{array}{l}
n \\
i
\end{array}\right) A^{i}=(1+A)^{n}
$$


By Theorem 2.1 and (11) we have $A \leq 1 / R$ which yields

$$
\operatorname{dim} H_{*}(M, \mathbb{Q}) \leq\left(1+\frac{1}{R}\right)^{n}
$$

as desired.

Corollary 1 follows from Theorem A and Corollary 2.2 if $M$ is rationally hyperbolic. If $M$ is rationally elliptic we always have $\operatorname{dim} H_{*}(M, \mathbb{Q}) \leq 2^{n}$.

Remark 2.3. It seems interesting to observe that from inequality (9) and Corollary 2.2 it follows that if $\left(M^{n}, g\right)$ is a closed simply connected formal manifold with $r \geq \delta g, \delta>0$, then

$$
\operatorname{dim} H_{*}(M, \mathbb{Q}) \leq\left[1+\exp \left(\pi h_{t o p}(g) \sqrt{\frac{n-1}{\delta}}\right)\right]^{n} .
$$

This suggests that the following smooth invariant of a manifold that admits a metric of positive Ricci curvature might be of interest:

$$
h_{r}(M):=\inf _{\{g: r(g) \geq g\}} h_{t o p}(g) .
$$

From the last inequality, if $M$ is formal and admits a metric of positive Ricci curvature we have

$$
\operatorname{dim} H_{*}(M, \mathbb{Q}) \leq\left[1+\exp \left(\pi \sqrt{n-1} h_{r}(M)\right)\right]^{n} .
$$

2.3. Proof of Corollary 2. In [9], Félix and Thomas point out the following corollary of Theorem 2.1:

Corollary 2.4. If $M^{n}$ is a closed $(p-1)$-connected formal manifold then

$$
R \leq\left(\frac{n}{p b_{p}}\right)^{1 / p}
$$

Corollary 2 follows right away from the Corollary 2.4 and Theorem A.

\section{Proof of Theorem B}

Let $S M \subset T M$ be the unit sphere bundle of $M$. We will consider the geodesic flow $\Phi_{t}$ of $g$ acting on $S M$. Given any $\theta \in S M$ we will denote by $c_{\theta}$ the geodesic with initial condition $\theta$. The expression $\theta=(x, v)$ will mean that $v \in T_{x} M$.

Recall that the metric $g$ on $M$ induces a metric on $T M$ (the Sasaki metric) and for any $\theta \in T M$ an orthogonal decomposition of $T_{\theta} T M$ into horizontal and vertical parts: $T_{\theta} T M=H_{\theta} \oplus V_{\theta}$ (the vertical space is of course the tangent space of the fiber). Recall also that the differential of $\Phi_{t}$ has a nice expression in geometric terms; given $\xi=\left(w_{1}, w_{2}\right) \in T_{\theta} T M, d_{\theta} \Phi_{t}(\xi)=\left(J_{\xi}(t), \dot{J}_{\xi}(t)\right)$, where $J_{\xi}$ is the Jacobi field along $c_{\theta}$ with initial conditions $J_{\xi}(0)=w_{1}$ and $\dot{J}_{\xi}(0)=w_{2}$. 
If $\theta=(x, v)$ we will denote by $S(\theta)$ the orthogonal complement of $(v, 0)$ in $T_{\theta} S M$. Equivalently, $S(\theta)$ is the orthogonal complement of the subspace spanned by $(v, 0)$ and $(0, v)$ in $T_{\theta} M$. It is easy to see that the subspaces $S(\theta)$ are invariant through the differential of the geodesic flow.

The proof of Theorem B will be based on Przytycki's inequality [28] for the topological entropy of the geodesic flow which we will describe now.

Given two real vector spaces with inner product $V$ and $W$ of the same dimension and a linear transformation $f: V \rightarrow W$ the expansion of $f, \operatorname{ex}(f)$, is the supremum over all non-trivial subspaces of $V$ of the absolute value of the determinant of $\left.f\right|_{V}$. An important (and trivial) property of the expansion is that given two linear maps $f$ and $g$ we have $\operatorname{ex}(f g) \leq \operatorname{ex}(f) \operatorname{ex}(g)$. This will be used below.

Przytycki's inequality is the following:

$$
h_{\text {top }}(g) \leq \liminf _{t \rightarrow \infty} \frac{1}{t} \log \int_{S M} \operatorname{ex}\left(d_{\theta} \Phi_{t}\right) d \theta .
$$

Here we consider $d_{\theta} \Phi_{t}$ as a map between $S(\theta)$ and $S\left(\dot{c}_{\theta}(t)\right)$.

Remark 3.1. We only need to use Przytycki's inequality although it is actually true that for a $C^{\infty}$ Riemanniann metric one has Mañés formula [22]:

$$
h_{\text {top }}(g)=\lim _{t \rightarrow \infty} \frac{1}{t} \log \int_{S M} \operatorname{ex}\left(d_{\theta} \Phi_{t}\right) d \theta=\lim _{T \rightarrow \infty} \frac{1}{T} \log \int_{M \times M} n_{T}(x, y) d x d y .
$$

Moreover, O. S. Kozlovski [19] has shown that Przytycki's inequality is an equality for arbitrary $C^{\infty}$ maps.

We will find our upper bound for the topological entropy by studying $\operatorname{ex}\left(d_{\theta} \Phi_{t}\right)$ for small values of $t$. Given any $\delta>0$ we have:

$$
h_{\text {top }}(g) \leq \liminf _{i \rightarrow \infty} \frac{1}{\delta i} \log \int_{S M} \operatorname{ex}\left(d_{\theta}\left(\left(\Phi_{\delta}\right)^{i}\right)\right) d \theta .
$$

Now suppose that for some $\delta>0$ and $\alpha>0$ we have that $\operatorname{ex}\left(d_{\theta} \Phi_{\delta}\right) \leq \alpha$ for all $\theta$. Then it follows that

$$
h_{\text {top }}(g) \leq \lim _{i \rightarrow \infty} \frac{1}{\delta i} \log \left(\alpha^{i} \operatorname{Vol}(S M)\right)=\frac{\log (\alpha)}{\delta} .
$$

We will get an estimate of the form $\alpha=1+\beta \delta+O\left(\delta^{2}\right)$. Then $\log (\alpha)=$ $\beta \delta+O\left(\delta^{2}\right)$ and since the last inequality holds for any $\delta>0$ we get

$$
h_{\text {top }}(g) \leq \beta \text {. }
$$

We will prove Theorem B by estimating $\beta$. Consider the polar decomposition of $d_{\theta} \Phi_{t}: d_{\theta} \Phi_{t}=O_{t}(\theta) L_{t}(\theta)$ where $O_{t}(\theta): S(\theta) \rightarrow S\left(\dot{c}_{\theta}(t)\right)$ is a linear isometry and $L_{t}(\theta)$ is a symmetric positive endomorphism of $S(\theta)$. Then $\operatorname{ex}\left(d \Phi_{t}\right)=$ $\operatorname{ex}\left(L_{t}\right)$. Of course, $L_{t}$ can be given explicitly: $L_{t}=\left(\left(d \Phi_{t}\right)^{*}\left(d \Phi_{t}\right)\right)^{1 / 2}$.

Consider the map $\mathcal{R}: S(\theta) \rightarrow S(\theta)$ which in the decomposition into horizontal and vertical parts is given by $\mathcal{R}\left(w_{1}, w_{2}\right)=\left(w_{2},-R\left(v, w_{1}\right) v\right)$, where $R$ is the curvature tensor and $\theta=(x, v) \in S M$. 
Lemma 3.2. $\left(\left(d \Phi_{\delta}\right)^{*}\left(d \Phi_{\delta}\right)\right)^{1 / 2}=I d+\frac{\delta}{2}\left(\mathcal{R}+\mathcal{R}^{*}\right)+O\left(\delta^{2}\right)$.

Proof. Given any $\theta=(x, v) \in S M$ let $c_{\theta}$ be the geodesic with initial condition $\theta$. Let $T_{t}$ be the parallel transport along $c_{\theta}$ from $T_{c_{\theta}(0)}$ to $T_{c_{\theta}(t)}$. Let $e_{1}, e_{2}, \ldots, e_{n-1}$ be an orthonormal basis of $\{v\}^{\perp} \subset T_{x} M$ by eigenvectors of the symmetric transformation $u \mapsto R(v, u) v$. Let $E_{i}$ be the parallel vector field along $c_{\theta}$ with initial condition $e_{i}$. Given $\xi \in T_{\theta} S M$ we can write $J_{\xi}(t)=\sum_{i=1}^{n-1} a_{i}(t) E_{i}(t)$ for some smooth functions $a_{i}$. Then,

$$
J_{\xi}(\delta)=\sum_{i=1}^{n-1}\left(a_{i}(0)+\delta a_{i}^{\prime}(0)\right) T_{\delta} E_{i}(0)+O\left(\delta^{2}\right)
$$

and since $\dot{J}_{\xi}(t)=\sum_{i=1}^{n-1} a_{i}^{\prime}(t) E_{i}(t)$, we have

$$
\dot{J}_{\xi}(\delta)=\sum_{i=1}^{n-1}\left(a_{i}^{\prime}(0)+\delta a_{i}^{\prime \prime}(0)\right) T_{\delta} E_{i}(0)+O\left(\delta^{2}\right) .
$$

Now, from the Jacobi equation, we get that $\sum_{i=1}^{n-1} a_{i}^{\prime \prime}(0) e_{i}=-R\left(v, J_{\xi}(0)\right) v$. Therefore

$$
\begin{aligned}
& \left(J_{\xi}(\delta), \dot{J}_{\xi}(\delta)\right)= \\
& \left(T_{\delta}\left(\sum_{i=1}^{n-1}\left(a_{i}(0)+\delta a_{i}^{\prime}(0)\right) e_{i}\right), T_{\delta}\left(\sum_{i=1}^{n-1} a_{i}^{\prime}(0) e_{i}-\delta R\left(v, J_{\xi}(0)\right) v\right)\right)+O\left(\delta^{2}\right),
\end{aligned}
$$

i.e.

$$
d \Phi_{\delta}=\left(T_{\delta}, T_{\delta}\right)(I d+\delta \mathcal{R})+O\left(\delta^{2}\right) .
$$

Since $T_{\delta}$ is orthogonal we get

$$
\left(d \Phi_{\delta}\right)^{*}\left(d \Phi_{\delta}\right)=I d+\delta\left(\mathcal{R}+\mathcal{R}^{*}\right)+O\left(\delta^{2}\right)
$$

and therefore,

$$
\left(\left(d \Phi_{\delta}\right)^{*}\left(d \Phi_{\delta}\right)\right)^{1 / 2}=I d+(\delta / 2)\left(\mathcal{R}+\mathcal{R}^{*}\right)+O\left(\delta^{2}\right) .
$$

From the lemma we obtain that $\operatorname{ex}\left(d \Phi_{\delta}\right)=\operatorname{ex}\left(I d+\frac{\delta}{2}\left(\mathcal{R}+\mathcal{R}^{*}\right)\right)+O\left(\delta^{2}\right)$; and we are left to compute $\operatorname{ex}\left(I d+\frac{\delta}{2}\left(\mathcal{R}+\mathcal{R}^{*}\right)\right)$. This is a positive definite symmetric endomorphism of $S(\theta)$ and therefore the expansion is the product of the eigenvalues which are greater than or equal to one, provided that there exists at least one eigenvalue greater than or equal to one.

We can compute the eigenvalues explicitly using the orthonormal basis of $S(\theta)$ given by:

$$
\left\{\left(e_{1}, e_{1}\right), \ldots,\left(e_{n-1}, e_{n-1}\right),\left(e_{1},-e_{1}\right), \ldots,\left(e_{n-1},-e_{n-1}\right)\right\} .
$$

Suppose that $R\left(v, e_{i}\right) v=\lambda_{i} e_{i}$. Then it is easy to check that

$$
\left(I d+(\delta / 2)\left(\mathcal{R}+\mathcal{R}^{*}\right)\right)\left(e_{i}, e_{i}\right)=\left(1+(\delta / 2)\left(1-\lambda_{i}\right)\right)\left(e_{i}, e_{i}\right)
$$

and $\left(I d+(\delta / 2)\left(\mathcal{R}+\mathcal{R}^{*}\right)\right)\left(e_{i},-e_{i}\right)=\left(1+(\delta / 2)\left(\lambda_{i}-1\right)\right)\left(e_{i},-e_{i}\right)$. 
If $\lambda_{i} \leq 1$ for all $i$ we get that

$\operatorname{ex}\left(I d+(\delta / 2)\left(\mathcal{R}+\mathcal{R}^{*}\right)\right)=\prod_{i=1}^{n-1}\left(1+(\delta / 2)\left(1-\lambda_{i}\right)\right)=1+(\delta / 2)(n-1-r(v))+O\left(\delta^{2}\right)$,

where $r(v)$ denotes, as before, the Ricci curvature in the direction of $v$.

Therefore if the sectional curvature of $g$ is bounded above by 1 we get, from the previous discussion, that

$$
h_{\text {top }}(g) \leq \frac{1}{2}\left(n-1-\min _{v \in S M} r(v)\right) .
$$

For a general $g$, let $k$ be the maximum of all the sectional curvatures. If $k>0$ the metric $g_{k}=k g$ has sectional curvature bounded above by one and from the previous observation we get that

$$
h_{t o p}\left(g_{k}\right) \leq \frac{1}{2}\left(n-1-\frac{\min _{v \in S M_{g}} r_{g}(v)}{k}\right) .
$$

But $h_{\text {top }}\left(g_{k}\right)=(1 / \sqrt{k}) h_{t o p}(g)$ and therefore we get

$$
h_{t o p}(g) \leq \frac{1}{2}\left(\sqrt{k}(n-1)-\frac{\min _{v \in S M} r(v)}{\sqrt{k}}\right) .
$$

This finishes the proof of Theorem B.

Remark 3.3. If the sectional curvature of $g$ is non-positive (i.e. if $k \leq 0$ ), we get that for any positive number $\rho$,

$$
h_{\text {top }}\left(g_{\rho}\right) \leq \frac{1}{2}\left(n-1-\frac{\min _{v \in S M_{g}} r_{g}(v)}{\rho}\right)
$$

and therefore,

$$
h_{t o p}(g) \leq \frac{1}{2}\left(\sqrt{\rho}(n-1)-\frac{\min _{v \in S M} r(v)}{\sqrt{\rho}}\right) .
$$

The sharpest inequality is obtained by taking $\rho=\frac{-\min _{v \in S M} r(v)}{n-1}$ and hence we have:

$$
h_{t o p}(g) \leq \sqrt{-(n-1) \min _{v \in S M} r(v)}
$$

The Bishop comparison theorem implies right away that if $r \geq-(n-1) g$ then $\lambda$, the exponential growth rate of volume of balls in the universal covering, satisfies

$$
\lambda \leq n-1 .
$$

But when the sectional curvature is non-positive, Manning proved [21] that $h_{t o p}(g)=\lambda$ and we recover inequality $(13)$. 


\section{References}

[1] I. Babenko, Topological entropy of geodesic flows on simply connected manifolds, and related problems, (Russian) Izv. Ross. Akad. Nauk Ser. Mat. 61 (1997), 57-74.

[2] I. Babenko, Analytic properties of Poincaré series of a loop space, (Russian) Mat. Zametki 27 (1980), 751-765.

[3] M. Berger, R. Bott, Sur les variétés à courbure strictement positive, Topology 1 (1962), 302-311.

[4] P. Deligne, P. Griffiths, J. Morgan, D. Sullivan, Real homotopy theory of Kähler manifolds, Invent. Math. 29 (1975), 245-274.

[5] Y. Félix, La dichotomie elliptique-hyperbolique en homotopie rationnelle, Astérisque 176 (1989), 187.

[6] Y. Félix, S. Halperin, Rational Lusternik-Schnirelmann category and its applications, Trans. Amer. Soc. 273 (1982), 1-37.

[7] Y. Félix, S. Halperin, J. C. Thomas, Elliptic spaces, Bull. Amer. Math. Soc. (N.S.) 25 (1991), 69-73.

[8] Y. Félix, S. Halperin, J. C. Thomas, Elliptic spaces II, Enseign. Math. 39 (1993), 25-32.

[9] Y. Félix, J. C. Thomas, The radius of convergence of Poincaré series of loop spaces, Invent. Math. 68 (1982), 257-274.

[10] M. H. Freedman, The topology of four-dimensional manifolds, J. Differential Geom. 17 (1982), 357-454.

[11] M. Gromov, Curvature, diameter and Betti number, Comment. Math. Helv. 56 (1981), 179-195.

[12] N. Grossman, The topology of taut Riemannian manifolds with positive risec pinching, J. Differentialial Geometry 3 (1969), 393-410.

[13] K. Grove, S. Halperin, Contributions of Rational Homotopy Theory to global problems in Geometry, Inst. Hautes Études Sci. Publ. Math. 56 (1982), 379-385.

[14] V. Guillemin, S. Sternberg, Symplectic techniques in physics, Cambridge University Press, 1984.

[15] M. J. Gursky, C. LeBrun, On Einstein manifolds of positive sectional curvature, Ann. Global Anal. Geom. 17 (1999) 315-328.

[16] K. Grove, S. Halperin, Dupin hypersurfaces, group actions and the double mapping cylinder, J. Differential Geometry 26 (1987), 429-459.

[17] S. Halperin, Spaces whose rational homology and De Rham homotopy are both finite dimensional, Astérisque, 113-114 (1984), 198-205.

[18] N.J. Hitchin, On compact four-dimensional Einstein manifolds, J. Differential Geom. 9 (1974), 435-442.

[19] O.S. Kozlovski, An integral formula for the topological entropy of $C^{\infty}$ maps, Ergodic Theory Dynam. Systems 18 (1998), 405-424.

[20] A. Manning, Topological entropy for geodesic flows, Ann. of Math. 110 (1979), 567-573.

[21] A. Manning, More topological entropy for geodesic flows, Dynamical Systems and Trubulence, Warwick 1980, D. Rand and L. S. Young eds, Lecture Notes in Math. 898 (1981), Springer, 243-249.

[22] R. Mañé, On the topological entropy of geodesic flows, J. Differential Geom. 45 (1997), 74-93.

[23] T. J. Miller, On the formality of $(k-1)$-connected compact manifolds of dimension less than or equal to $4 k-2$, Illinois J. Math. 23 (1979), 253-258.

[24] J. Milnor, Morse Theory, Ann. of Math. Studies 51, Princeton Univ. Press: Princeton, NJ, 1969.

[25] J. Neisendorfer, T.J. Miller, Formal and coformal spaces, Illinois J. Math. 22 (1978), 565-580.

[26] G. P. Paternain, Geodesic Flows, Progress in Mathematics 180, Birkhäuser, 1999. 
[27] G. P. Paternain, On the topology of manifolds with completely integrable geodesic flows, Ergodic Theory Dynamical Systems 12 (1992), 109-121.

[28] F. Przytycki, An upper estimation for the topological entropy of diffeomorphisms, Invent. Math. 59 (1980), 205-213.

[29] J. P. Serre, Groupes d'homotopie et classes de grupes abéliens, Ann. of Math. 58 (1953) 258-294.

[30] Y. Yomdin, Volume growth and entropy, Israel J. Math. 57 (1987), 285-300.

Cimat, A.P. 402, 36000, Guanajuato. Gto., México.

E-mail address: paternain@cimat.mx

Cimat, A.P. 402, 36000, Guanajuato. Gto., México.

E-mail address: jimmy@cimat.mx 